\title{
BREEDING BIRD COMMUNITIES IN A NATURALLY FRAGMENTED FOREST ECOSYSTEM IN GRAND TETON NATIONAL PARK
}

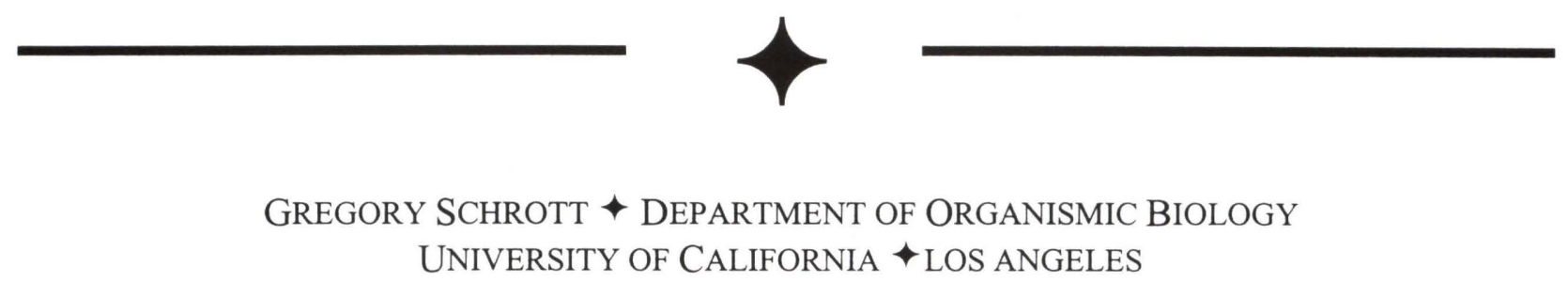

\section{$\uparrow$ RESEARCH SUMMARY}

This project examined the bird species breeding in the morainal forests on the valley floor in Grand Teton National Park. These forests are very patchily distributed and range in size from less than 1 hectare to over 700 hectares, allowing for a unique opportunity to study the responses of the local bird species to a forest system that has been fragmented for centuries through natural processes. This information can be useful for predicting the potential long-term impacts of human-caused forest fragmentation on bird populations in western North America. Until quite recently very little was known of the tolerances of western forest bird species to habitat fragmentation and this project could represent an important step towards understanding their needs in this regard.

Full-time data collection on this project began in the summer of 1997 and continued in the summers of 1998 and 1999. Point count bird censuses were conducted at 60 permanent survey plots in forest patches of various size, as well as in unfragmented pine forests around the valley floor. These bird counts were used to construct incidence curves which demonstrate the preferences of each species in the community for various sizes of forest patch. Four basic patterns of incidence were detected: (1) birds which are common across all sizes of forest patch (e.g.: Dark-eyed Junco), (2) birds which are most common in medium-sized (10-25 hectares) forest patches (e.g.: Warbling Vireo), (3) bird species which are found in modest numbers in medium-sized forest patches, but are most common in unfragmented forest (e.g.: Ruby-crowned Kinglet), and (4) birds which are found almost exclusively in unfragmented forest (e.g.: Hermit Thrush). By examining the data from all 3 years, it is possible to examine populations of birds which fluctuated a great deal during the study and therefore show which patches a species preferred to settle in when population pressures were higher and lower, therefore indicating which patches are indeed most desirable for a given species rather than merely tolerable.

In addition to bird censuses, during the summer of 1998, vegetation surveys of all 60 census sites were done in order to better determine the finalscale habitat selection criteria for the various species beyond simple patch size. While the analyses of these data is still ongoing, it is evident that patch size does effect the character of the forest patches in that trees in small patches tend to grow much more densely and do not tend to be as large as those in larger patches. There also tends to be a much higher proportion of lodgepole pine than fir or spruce in these small patches. It is evident that some of the patterns in forest patch size selection in birds are related to the qualitative nature of the different sizes of forest patch.

The field data collection for this project is essentially completed. While some sites may be revisited again in the future if the opportunity arises, there will probably be no work done in these patches during the 2000 field season. 